\title{
Productivity Calculation for Fractured Wells in Low Permeability Gas Reservoirs
}

\author{
Hua Cai, Guohua Zhang, Yangfan Li \\ CNOOC Ltd-Shanghai, China
}

\begin{abstract}
PH gas field, which located in East China Offshore, has the characteristic: Prematurely Senile Appearance. That means it is currently in the middle period of development, but has many features of the later period of the gas field's life. In order to compensate the reduction of gas production, the development of low permeability gas reservoirs has been carried out these years. Low permeability gas reservoirs which distributed in the lower stratum have the permeability ranges between $0.3 \mathrm{mD} \sim 1 \mathrm{mD}$. Developing practice shows that the reservoirs with permeability lower than $1 \mathrm{mD}$ haven't natural productivity to maintain regular production. As a result, researches and practices of reconstruction and fracturing had been implemented during the past years. In recent researches, calculation methods related to productivity, reservoir selection and fracturing design etc. were developed and applied, while the equations and analysis methods in accordance with the practice were presented in this paper.
\end{abstract}

\section{Keywords-low permeability; fracturing; fractured wells}

\section{INTRODUCTION}

There are two types of reservoirs in $\mathrm{PH}$ gas field: Conventional reservoirs distributed in the upper stratum; Unconventional reservoirs with low porosity and low permeability distributed in the lower stratum. In order to improve the productivity of the whole gas field, CNOOC has implemented series of fracturing reconstruction researches and practices on the development of the low permeability gas reservoir.

The choice of methods on production improvement is related with the properties of the gas reservoirs. Without considering the influence of natural fracture and skin, permeability of the reservoir is the most important factor on the production improvement. At present, fracturing is the most appropriate method to improve the permeability, and it could improve the porosity at same time.

Generally, the requirement of Communication Acreage between the reservoir and wellbore will increase with the decrease in permeability of the reservoir. Typically, since the permeability of the reservoir decreases massively, the improvement of the Communication Acreage for the directional well through traditional way will especially difficult to take place, therefore, the Horizontal Well Fracturing Technology would be chosen for consideration.

CNOOC has implemented series of fracturing reconstruction researches and practices on low permeability gas reservoirs. The practices have gotten the staged achievements, and researches and practices on fracturing are still maintaining at present.

\section{MATHEMATICS}

The factors about production before and after fracturing in directional well are already existed as actual data from the production history. Therefore, the factors about new wells after fracturing could be calculated by the formulas which related to the experience.

\section{A. Productivity Statistics for Directional Wells}

After fracturing on directional well, the productivity $Q_{g}$, could be expressed as follow:

$$
Q_{g}^{\prime}=a_{1} \cdot\left(S_{g} \cdot \sqrt{K / \Phi}\right)^{b_{1}} \cdot H \cdot \Delta P
$$

Wher $Q_{g}$ is the productivity of directional wells after fracturing, $\mathrm{m}^{3} / \mathrm{D} ; S_{g}$ is the gas saturation; $K$ is the permeability, $\mathrm{mD} ; \stackrel{\Phi}{\Phi}$ is the porosity; $H$ is the average thickness of the reservoir, $\mathrm{m} ; \Delta p$ is the drawdown pressure, $\mathrm{MPa} ; a_{1}$ and $b_{1}$ are constant.

Revising the skin, the productivity $Q_{g}$ could be expressed as follow:

$$
Q_{g}=Q_{g}^{\prime} \cdot\left(\ln \frac{r_{e}}{r_{w}}+S\right) / \ln \frac{r_{e}}{r_{w}}
$$

Where $Q_{g}$ is the productivity of the well after correction of the skin, $\mathrm{m}^{3} / \mathrm{D} ; S$ is skin factor, constant; $r_{e}$ is the radius of the drainage area, $\mathrm{m} ; r_{w}$ is the radius of the well bore, $\mathrm{m}$.

Take formula (1) into formula (2), the $Q_{g}$ could be expressed as follow:

$$
Q_{g}=a_{1} \cdot\left(S_{g} \cdot \sqrt{K / \Phi}\right)^{b_{1}} \cdot H \cdot \Delta P \cdot\left(\ln \frac{r_{e}}{r_{w}}+S\right) / \ln \frac{r_{e}}{r_{w}}
$$

By using the formula (3) after revising the skin, the result is the initial productivity after fracturing in directional well.

\section{B. Productivity Simulation Formula for Directional Wells}

Productivity simulation formula is related with the core experiments. The gas production under different pressure could be simulated by using the simulation of reservoir state which includes: Initial water saturation, Salinity of Formation Water, Overlying Pressure, Reservoir Pressure, Reservoir Temperature, etc.

Based on the experimental data, the relationship between the permeability and average thickness could be expressed as follow:

$$
Q_{g}=\left[a_{2} \cdot K \cdot\left(P_{r}^{2}-P_{w f}{ }^{2}\right)-b_{2}\right] \cdot H
$$


Where $P_{r}$ is the initial pressure of the reservoir, MPa; $P_{w f}$ is the bottom-hole flowing pressure, $\mathrm{MPa} ; a_{2}$ and $b_{2}$ are constant.

\section{Productivity Formula for Horizontal Wells}

Since there is no actual production data after fracturing in horizontal wells, the formula for productivity calculation in horizontal wells should be deduced mathematically

There are two sections of pressure drop through process of the fluid which flow into the wellbore from reservoir: (1) pressure drop between the fracture and the horizontal section; (2) pressure drop between the horizontal section and the wellbore.

Based on the above assumptions, without consideration of the skin effect, the two sections of pressure drop could be calculated separately.

With consideration of pressure-sensitive effect, pressure drop between the fracture and the horizontal section could be expressed as follow:

$$
\Delta p_{1}=-\frac{2}{\alpha_{k}} \ln \left[1-\frac{Q_{i} \mu \alpha_{k} X_{f}}{K_{i} W H}\right]
$$

Where $\alpha_{k}$ is the coefficient of pressure-sensitive effect, $\mathrm{MPa}^{-1} ; \mu$ is the viscosity, MPa.s; $H$ is the thickness of the reservoir, $\mathrm{m} ; k_{i}$ is the permeability of the reservoir, $\mu \mathrm{m}^{2} ; Q_{i}$ is the production of each fracture, $\mathrm{m}^{3} / \mathrm{d} ; X_{f}$ is the half length of the fracture, $\mathrm{m} ; W$ is the width of the fracture, $\mathrm{m}$.

Pressure drop between the horizontal section and the wellbore could be expressed as follow:

$$
\Delta p_{2}=-\frac{1}{2 \pi} \cdot \frac{Q_{i} \mu}{K_{f} w}\left[\ln \frac{H}{2 r_{w}}-\frac{\pi}{2}\right]
$$

Where $K_{f}$ is the permeability of the fracture, $\mu \mathrm{m}^{2} ; r_{w}$ is the radius of the wellbore, $\mathrm{m}$.

Pressure drop of the whole production system could be expressed as follow:

$$
\Delta p=p_{r}-p_{w f}=\Delta p_{1}+\Delta p_{2}
$$

Where $P_{r}$ is the initial pressure of the reservoir, $\mathrm{MPa}$; $P_{w f}$ is the bottom-hole flowing pressure, MPa.

According to formula (5), (6) and (7), pressure drop of the whole production system could be expressed as follow:

$$
\Delta p=-\frac{2}{\alpha_{k}} \ln \left[1-\frac{Q_{i} \mu \alpha_{k} X_{f}}{k_{i} w H}\right]-\frac{1}{2 \pi} \cdot \frac{Q_{i} \mu}{k_{f} w}\left[\ln \frac{h}{2 r_{w}}-\frac{\pi}{2}\right]
$$

Using the formula (8), and the drawdown pressure is equal to the pressure drop of the whole production system $\Delta p$, by fixing the drawdown pressure, it is a given number. The production $Q_{i}$ of one fracture could be calculated through Newton iteration method.

Assuming the number of fractures is $N$, the total production under the given $\Delta p$ could be expressed as follow:

$$
Q=\sum_{i=1}^{N} Q_{i}
$$

\section{RESULTS}

\section{Productivity of Directional Well}

As can be seen in the Figure 1 , the constant $a_{1}$ and $b_{1}$ in equation (3) are 0.36 and 2.97.

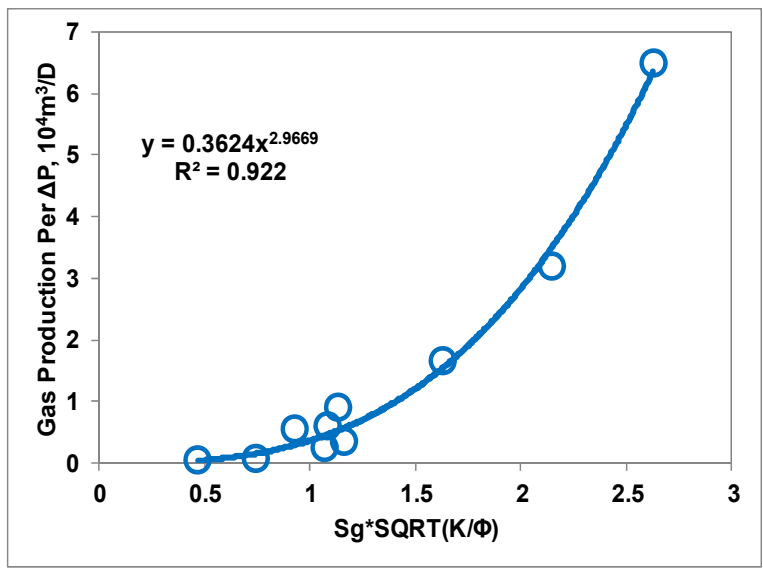

Figure 1. Calculation of the Production by Formula (3)

As can be seen in the Figure 2, the constant $a_{2}$ and $b_{2}$ in equation (4) are 0.0012 and 0.25 .

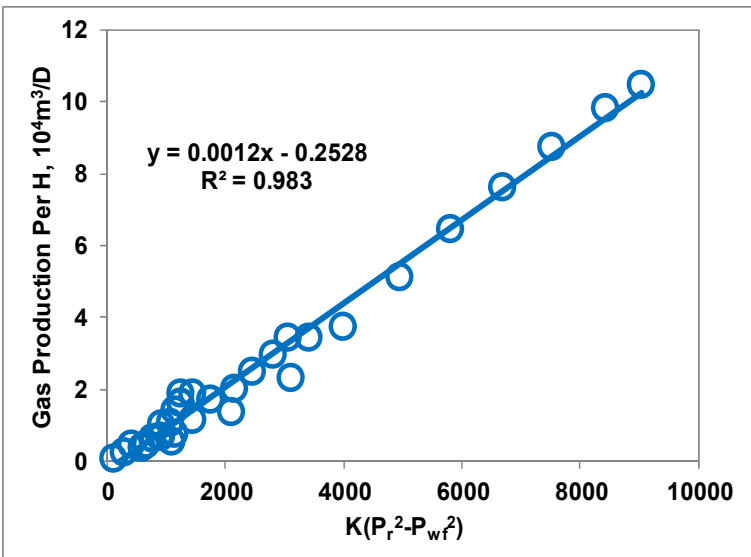

Figure 2. Calculation of the Production by Formula (4)

Comparison of predicted and actual results, as figure 3 , each formula has a precise prediction result on productivity for fractured directional wells. 


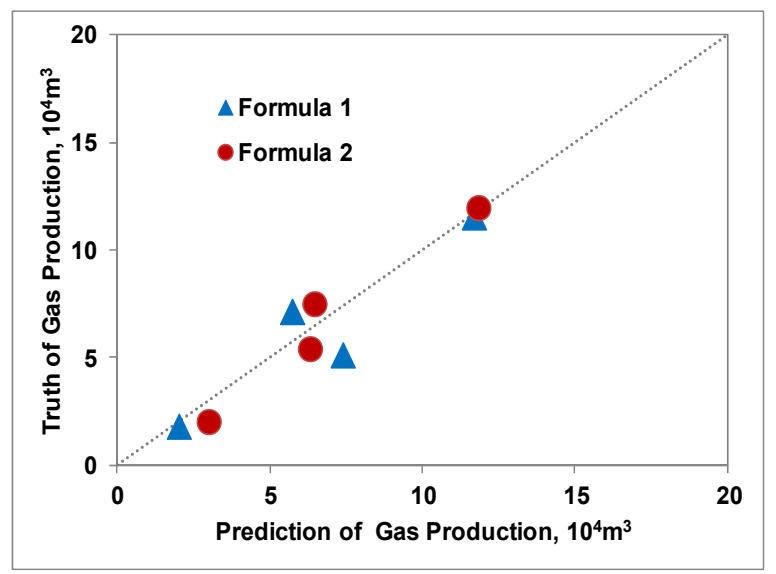

Figure 3. Comparison of Predicted and Actual Result of the Production

\section{E. Productivity of Horizontal Well}

The production calculated by the formula under different permeability is shown as Figure 4, and the production under different drawdown pressure is shown as Figure 5

As can be seen from the two Figures 4 and 5, due to the effect of the pressure sensitive, the results calculated by the formula related with horizontal wells are lower than the result from numerical simulation

As can be seen from Figures 5, since the drawdown pressure increases, the results of numerical calculations increases straightly, but the results of formula inflected. The interpretation is that, as the drawdown pressure increases, pressure sensitive effect would influence more on the production, as a result, the inflected curve is more reasonably and more preciously

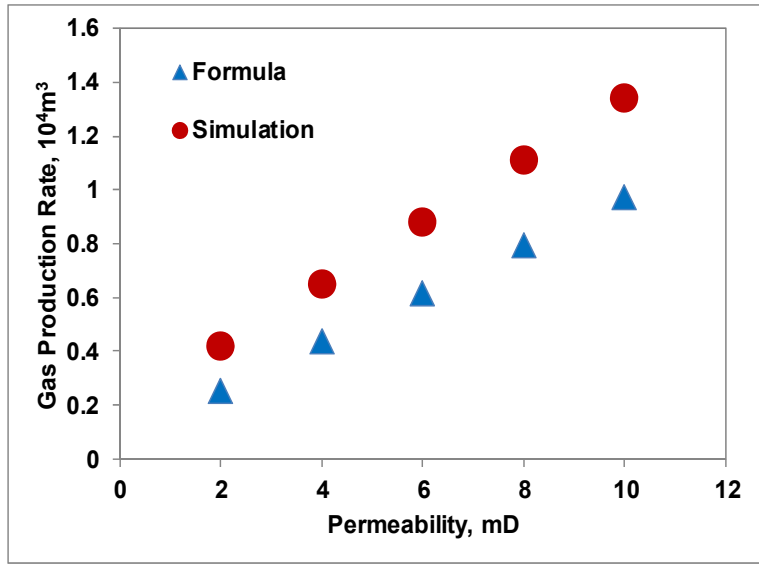

Figure 4. Production Calculation by Formula (8) and (9)

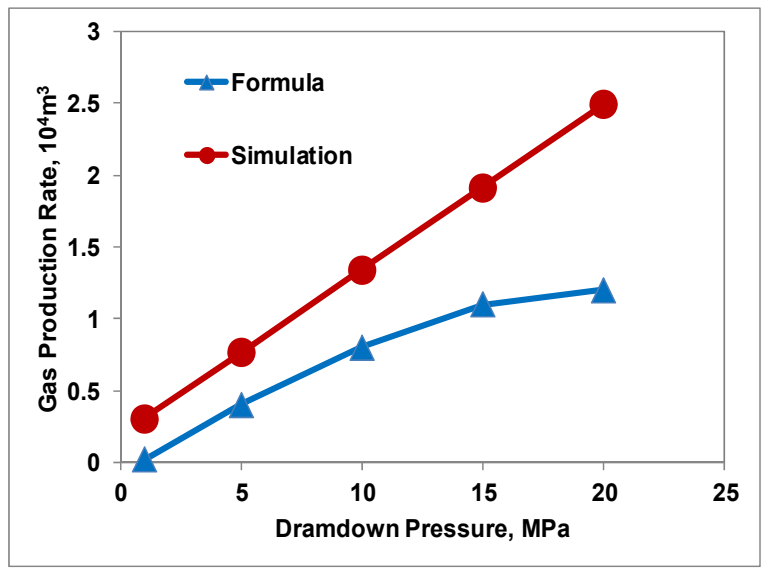

Figure 5. Production Calculation by Formula (8) and (9)

\section{SENSITIVITY ANALYSIS}

The sensitivity analysis of the reservoir pressure on permeability and porosity is shown in Figure 6

Sensitivity Analysis is a method which indicates that: research on the effect of main control parameters in the reservoir and help to making more effective decisions

The result of the Sensitivity Analysis indicates that: the most sensitive parameter is the $\Phi$; secondly, the Gas Saturation $\left(S_{g}\right)$; thirdly, the Thickness $(H)$ and the Acreage $(A)$; lastly, the gas deviation factor $\left(Z_{i}\right)$

According to the above analysis, the improvement on porosity and permeability is the most important method to improve the recovery in low permeability gas reservoirs.

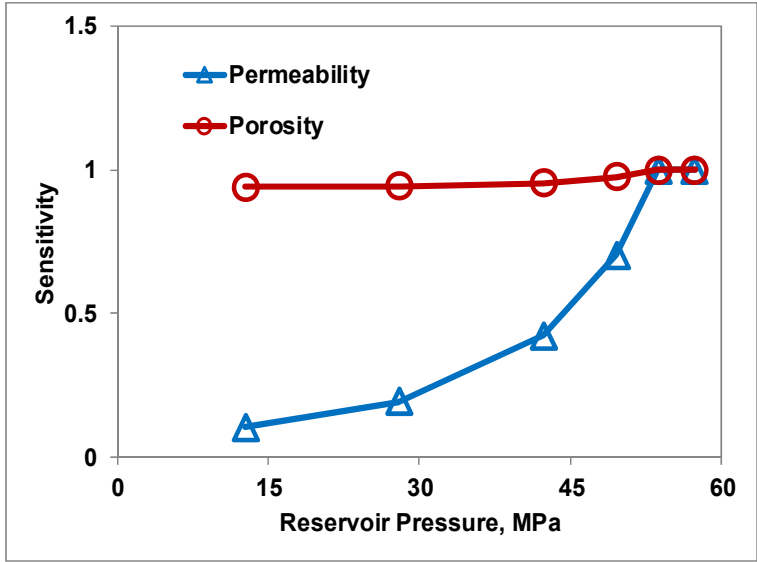

Figure 6. Sensitivity Analysis of Reservoir Pressure

\section{UNCERTAINTY ANALYSIS}

Uncertainty Analysis, which is a method commonly used in decision analysis, could help to reduce the negative effect of uncertainty on the calculation of production results and economic budget.

In the process of the permeability interpretation, the well logging interpretation and core analysis have a poor correlation, especially in the Tight Sandstone. The solution 
to this problem is: calculating and matching the permeability by core experiment individually on each well. The application of the formula calculated by one well on other wells also has a poor accuracy, especially in the Tight Sandstone under the environment of Underwater Distributary Channel Sedimentary. Due to the inevitable errors, and the significant effect of the permeability on Fracturing, DFIT Small Test was advised to implement in order to reduce the risk of fracturing.

\section{CONCLUSIONS}

The following conclusions could be drawn according to the results obtained in this study:

According to the result of sensitivity analysis, the porosity and permeability are the most important sensitive factors to improve the recovery in low permeability gas reservoirs, as a result, fracturing construction, is widely chose as the priority method.

Generally, the requirement of Communication Acreage between the reservoir and wellbore will increase with the decrease in permeability of the reservoir. Since the permeability of the reservoir decreases massively, the improvement of the Communication Acreage for the directional wells through traditional way will especially difficult to take place, therefore, the Horizontal Well Fracturing Technology would be chosen for consideration.

By using the parameters by Comprehensive Logging Interpretation, the factor of $S g \Phi H$ could be used for selection of the layers and wells for fracturing.

The formulas which were deduced from fractured directional wells, have acceptable accuracy, and could be used in calculation and prediction on new directional wells which would be fractured in the future.

Uncertainty analysis concluded that, the parameters and prediction results have inevitable error. The accuracy of prediction could be calculated after DFIT Small Test, and it still needs further research.

\section{ACKNOWLEDGEMENTS}

The author Hua Cai would like to acknowledge the colleagues for their hard work and contribution in this research. The author Guohua Zhang would like to acknowledge SINOPEC Ltd-Shanghai and Halliburton LtdChina for the technical references. This paper is based on many reports and researches of many colleagues in Research Institute. All the authors wish to acknowledge the leaders of CNOOC Ltd-Shanghai for the support and permission to publish this paper.

\section{REFERENCES}

[1] Robert F, M Y Soliman, Mike Vennes. Evaluating the Effects of Well Type Selection and Hydraulic Fracture Design on Recovery for Various Reservoir Permeably using a Numerical Reservoir Simulator. SPE 130108-MS, presented at SPE EUROPEC/EAGE Annual Conference and Exhibition, Barcelona, Spain, 14-17 June, 2010.

[2] Zhenbao Mu, Xiangchun Yuan, Xiaomin Zhu. The Calculating Method of Horizontal Wells with Hydraulic Fractures for Low Permeability Reservoirs. Geoscience (China) April 2009; Vol. 23, No. 2.

[3] Daoyong Yang, Qi Zhang, Ling Fan. Inflow performance of horizontal wells in naturally fractured reservoirs. Journal of the University of Petroleum 1999; 23 (6): 44-47.

[4] Giger F M. Horizontal Wells Production Techniques in Heterogeneous Reservoirs. SPE 13710-MS, presented at SPE Middle East Oil Technical Conference and Exhibition, Bahrain, March 11-14, 1985.

[5] Joshi S D. A Review of Horizontal Well and Drainhole Technology. SPE 16868-MS, presented at SPE Annual Technical Conference and Exhibition, Dallas, Texas, 27-30 September, 1987.

[6] Hujun Li, Zhengqi Jia, Zhaosheng Wei. A New Method to Predict Performance of Fractured Horizontal Wells. SPE 37051-MS, presented at SPE International Conference on Horizontal Well Technology, Calgary, Alberta, Canada, 18-20 November, 1996.

[7] Guppy K H, Kumar S, Kagawan V D. Pressure-Transient Analysis for Fractured Wells Producing at Constant Pressure. SPE 13629-PA. SPE Formation Evaluation 1988; Vol. 3, No. 1: 169-178. http://dx.doi.org/10.2118/13629-PA

[8] Naceur, Kamel Ben, Touboul, Eric. Mechanisms Controlling FractureHeight Growth in Layered Media. SPE 16433-PA. SPE Production Engineering 2013; Vol. 5, NO. 2: 142-150. http://dx.doi.org/10.2118/16433-PA

[9] Valko P P, Oligney R E, Economides M J. High permeability fracturing of gas wells. Petroleum Engineer International 1998; 71 (1): 75-88.

[10] Hemanta, Mukherjee, Michale J Economides. A parametric comparison of horizontal and vertical well performance. SPE18303PA. SPE Formation Evaluation 1988: 209 216. 